\title{
Cirurgia de revascularização do miocárdio associada a endarterectomia de carótida
}

Januário M. SOUZA*, Marcos F. BERLINCK*, Paulo Agostinho Fernandes de OLIVEIRA*, Rogério Petrassi FERREIRA* ${ }^{*}$ Ricardo MAZZIERI*, Sérgio Almeida de OLIVEIRA*

SOUZA, J. M.; BERLINCK, M. F.; OLIVEIRA, P. A. F.; FERREIRA, R. P.; MAZZIERI, R.; OLIVEIRA, S. A. Cirurgia de revascularizaçăo do miocárdio associada a endarterectomia de carótida. Rev. Bras. Cir. Cardiovasc., 10 (1): 43-49, 1995.

RESUMO: A oportunidade para realizaçāo de endarterectomia de carótida $(E C)$ e revascularização do miocárdio (RM) para doença concomitante ainda é controversa. Entre 1979 e 1994, 10940 pacientes foram operados para revascularização do miocárdio. Cirurgia combinada RM/EC foi realizada em $46(0,43 \%)$ pacientes, no mesmo período. A idade variou de 48 a 76 anos, média de 65,2 anos, sendo $34,7 \%(16 / 46) \mathrm{com}$ 70 anos ou mais; $80,4 \%$ eram do sexo masculino. Infarto do miocárdio prévio estava presente em $23(50 \%)$ pacientes. Diabetes mellitus em $10(21,7 \%)$, insuficiência renal crônica em $5(10,8 \%) ; 29(63 \%)$ estavam em classe funcional III ou IV para angina; havia ICC em 4 (8,6\%), lesão obstrutiva significativa em $9(19,5 \%)$ pacientes em tronco da coronária esquerda; 5 pacientes estavam sendo reoperados para RM e 1 para RM e EC. Dos 25 pacientes com lesão carotídea bilateral, 4 tinham uma artéria carótida interna ocluída. Vinte e três pacientes tinham tido isquemia cerebral transitória (ICT) e 2 pacientes acidente vascular cerebral (AVC) com seqũela permanente; 21 não tinham manifestação neurológica. Endarterectomia de carótida foi realizada na carótida esquerda em 23, na direita em 14 e bilateral em 9 . A RM foi realizada com pontes de safena e artéria torácica interna (ATI), variando de 1 a 4 pontes, com média de 2,8 pontes por paciente; 11 receberam ATI. A mortalidade imediata foi de 8,6\% (4/46); AVC permanente năo ocorreu no trans-operatório. Três dos 4 óbitos ocorreram em pacientes com idade igual ou superior a 70 anos. A EC foi realizada após preparo $e$ heparinização do paciente para circulação extracorpórea (CEC). Nos 4 pacientes que apresentavam oclusão da artéria carótida interna contralateral a EC foi realizada após o estabelecimento da CEC com hipotermia sistêmica $25^{\circ} \mathrm{C}$. Em nenhum caso foi utilizado shunt carotídeo. Nos casos de lesōes cirúrgicas bilaterais, 0 lado com lesão de maior risco foi operado juntamente com a RM, sendo a outra carótida operada em segundo tempo (em torno de uma semana). Considerando que nenhum dos pacientes apresentou AVC transoperatório, achamos ser a conduta adequada para essa associação de lesões.

DESCRITORES: Miocárdio, revascularização, cirurgia. Carótida, endarterectomia.

\section{INTRODUÇÃO}

Ainda não está definida a melhor conduta para o tratamento de pacientes portadores de obstrução de artérias coronárias associada a obstrução de carótidas. A incidência de aterosclerose carotídea varia na dependência de: grupo etário, sexo, taba- gismo e hipertensão arterial sistêmica. Tem sido encontrada em $6 \%$ a $16 \%$ dos pacientes candidatos a revascularização do miocárdio $2,7,8,15$. As publicações nem sempre definem de maneira adequada a extensão da doença carotídea, pois é comum, em pacientes portadores de sopro cervical, não se encontrar obstrução arterial ${ }^{15}$. Os pacientes que

Trabalho realizado no Serviço de Cirurgia Cardiovascular do Prof. Sérgio Almeida de Oliveira - Hospital da Beneficência Portuguesa. São Paulo, SP, Brasil.

Apresentado ao $22^{\circ}$ Congresso Nacional de Cirurgia Cardiaca. Brasilia, DF, 30 de março a $1^{2}$ de abril, 1995.

* Do Serviço de Cirúrgia Cardiovascular do Prof. Sérgio Almeida de Oliveira.

Endereço para correspondência: Januário M. Souza. Rua Pará, 40, $3^{\circ}$ andar. CEP (011) 255.1787 São Paulo, SP, Brasil. Tel. (011) 255.1787, Fax: (011) 257-9431. 
SOUZA, J. M.; BERLINCK, M. F.; OLIVEIRA, P. A. F.; FERREIRA, R. P.; MAZZIERI, R.; OLIVEIRA, S. A. - Cirurgia de revascularização do miocárdio associada a endarterectomia de carótida. Rev. Bras. Cir. Cardiovasc., 10 (1): $43-49,1995$.

têm lesão carotídea significativa e, principalmente, lesões bilaterais e quadro de isquemia cerebral transitória ou acidente vascular cerebral permanente, apresentam maior risco de complicação neurológica, se submetidos a cirurgia de revascularização do miocárdio $12,14,16$. BERNHARD et alii ${ }^{4}$ foram os primeiros a propor o tratamento cirúrgico combinado para lesões de artérias coronárias e carótidas. A partir dessa época, muitos trabalhos têm sido publicados, com resultados variados $12,14,17,19$. Quando a lesão da artéria carótida é acentuada, o risco de lesão cerebral durante cirurgia cardiaca é maior não só no pós-operatório imediato, mas, principalmente, na evolução a médio e longo prazos ${ }^{3}$.

\section{CASUÍSTICA E MÉTODOS}

Entre janeiro de 1979 e novembro de 1994, 10940 pacientes foram submetidos a cirurgia de revascularização do miocárdio. Nesse mesmo período foram realizadas operações combinadas para revascularização do miocárdio e endarterectomia de carótida em $46(0,43 \%)$ pacientes.

TABELA 1

ENDARTERECTOMIA DE CARÓTIDA ASSOCIADA A
REVASCULARIZAÇÃOMIOCÁRDICA
$1979-1994$
$N=46$

\begin{tabular}{|c|c|c|c|}
\hline \multicolumn{3}{|l|}{ DADOS } & $\%$ \\
\hline \multicolumn{2}{|c|}{$\begin{array}{l}\text { Sexo Masculino } \\
\text { Idade }(48-76 a)\end{array}$} & 37 & 80,4 \\
\hline \multicolumn{2}{|c|}{ Média } & 65,2 & - \\
\hline$\geq 70$ anos & & 16 & 34,7 \\
\hline \multicolumn{2}{|l|}{ Diabetes } & 10 & 21,7 \\
\hline \multicolumn{2}{|l|}{ IRC } & 5 & 10,8 \\
\hline \multicolumn{2}{|l|}{ HAS } & 37 & 80,4 \\
\hline \multicolumn{2}{|c|}{ Infarto do Miocárdio Prévio } & 23 & 50,0 \\
\hline \multicolumn{2}{|c|}{ Lesão TCE } & 9 & 19,5 \\
\hline \multicolumn{2}{|l|}{$\begin{array}{l}\text { ICC (IV) } \\
\text { Angina }\end{array}$} & 4 & 8,6 \\
\hline \multicolumn{2}{|l|}{$1-11$} & 17 & 37 \\
\hline \multirow{2}{*}{\multicolumn{2}{|c|}{$\begin{array}{l}\text { III - IV } \\
\text { Neurológico }\end{array}$}} & 29 & 63 \\
\hline & & & \\
\hline \multicolumn{2}{|c|}{ Assintomático } & 21 & 45,6 \\
\hline \multicolumn{2}{|c|}{ Isquemia Cerebral Transitória } & 23 & 50,0 \\
\hline \multicolumn{3}{|c|}{ AVC com seqũela } & 4,4 \\
\hline \multicolumn{4}{|c|}{ № Enxertos Ao-Coronarianos } \\
\hline \multirow{4}{*}{$\begin{array}{c}\text { Média } \\
1 \\
2 \\
3 \\
4\end{array}$} & 2,8 & & \\
\hline & 1 & (n) & \\
\hline & 11 & & \\
\hline & $\begin{array}{l}29 \\
5\end{array}$ & & \\
\hline \multicolumn{3}{|c|}{$\begin{array}{l}\text { IRC - Insuficiência Renal Crônica } \\
\text { HAS - Hipertensão Arterial Sistêmica } \\
\text { ICC - Insuficiência Cardiaca Congestiva } \\
\text { AVC - Acidente Vascular Cerebral }\end{array}$} & \\
\hline
\end{tabular}

A idade dos pacientes variou de 48 a 76 anos, com média de 65,2 anos; $34,7 \%(16 / 46)$ tinham 70 anos ou mais e $80,4 \%$ eram do sexo masculino. Infarto do miocárdio prévio estava presente em 23 $(50 \%)$, diabetes em $10(21,7 \%)$, insuficiência renal crônica em 5. Quanto à classe funcional (CF) 29 (63\%) pacientes estavam em CF III ou IV para angina e 4 tinham insuficiência cardíaca congestiva; $19,5 \%$ dos pacientes tinham lesão do tronco da coronária esquerda e 5 estavam sendo reoperados para revascularização do miocárdio e 1 para revascularização do miocárdio e endarterectomia de carótida (Tabela 1).

A quase totalidade dos pacientes tinha indicação primária para revascularização do miocárdio e a lesão arterial carotídea foi diagnosticada por achado de sopro cervical em 21 pacientes neurologicamente assintomáticos, em 23 pacientes com isquemia cerebral transitória e em $2 \mathrm{com}$ acidente vascular cerebral com seqüela permanente (Figura 1). Os pacientes com sopro foram submetidos a oculopletismografia ou estudo ultra-sonográfico e, quando havia sinais de lesão acima de $50 \%$ ou placas ulceradas, eram submetidos a arteriografia convencional ou digital, da aorta ascendente, arco aórtico e vasos braquiocefálicos. A indicação cirúrgica sempre foi decidida após estudo arteriográfico. Em 25 pacientes havia obstrução carotídea bilateral, sendo, em 4, oclusão de uma das carótidas (Tabela 2).

\section{TÉCNICA CIRÚRGICA}

Após monitorização habitual para operação com circulação extracorpórea, o preparo do campo operatório incluía o pescoço do lado a ser operado e

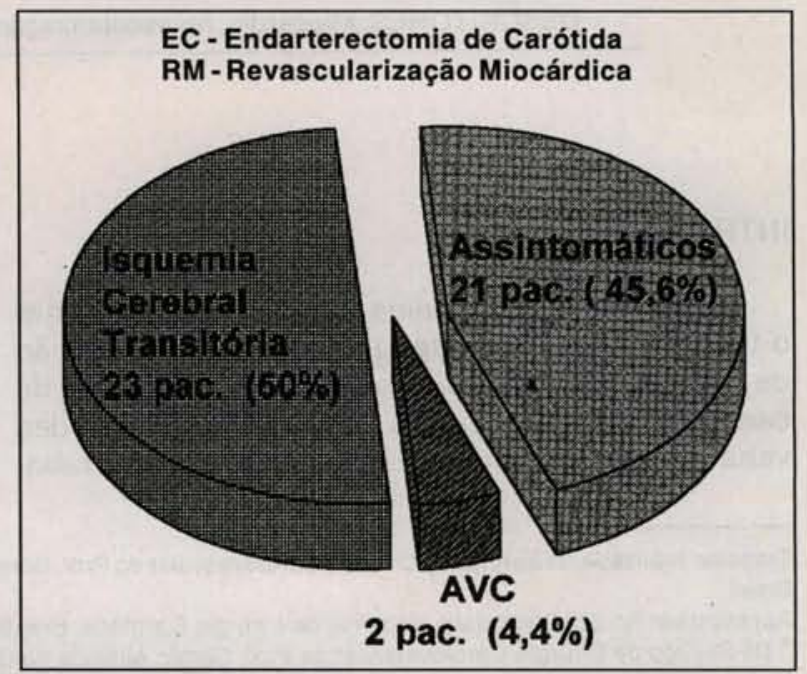

Fig. 1 - Situação neurológica dos pacientes submetidos a EC associada a RM 
SOUZA, J. M.; BERLINCK, M. F.; OLIVEIRA, P. A. F.; FERREIRA, R. P.; MAZZIERI, R.; OLIVEIRA, S. A. - Cirurgia de revascularização do miocárdio associada a endarterectomia de carótida. Rev. Bras. Cir. Cardiovasc., 10 (1): $43-49,1995$.

TABELA 2

ENDARTERECTOMIA DE CARÓTIDA ASSOCIADA A REVASCULARIZAÇĀOMIOCÁRDICA $1979-1994$

\begin{tabular}{llc}
\hline ANATOMIA DA CARÓTIDA & N & $\%$ \\
\hline Obstruçāo Unilateral & 21 & 45,6 \\
Obstruçāo Bilateral $^{*}$ & 25 & 54,4 \\
\hline
\end{tabular}

* 4 Pacientes tinham oclusão de uma das carótidas

a incisão cervical realizada concomitante à retirada do segmento de veia safena. A toracotomia era feita após dissecção da artéria carótida. Após dissecção de artéria torácica interna, exposição do coração e aorta ascendente, era administrada heparina $(4 \mathrm{mg} /$ $\mathrm{kg}$ de peso). A endarterectomia foi realizada em 42 pacientes, antes de iniciada a circulação extracorpórea; não se usou shunt interno. À retirada do ateroma, seguiu-se sutura direta da arteriotomia, exceto em 3 pacientes em que se usou retalho de veia safena.

Após a endarterectomia da carótida, era estabelecida a circulação extracorpórea com hipotermia moderada $\left(30^{\circ} \mathrm{C}\right)$ e realizada a revascularização do miocárdio; para proteção miocárdica foi utilizada solução cardioplégica (Buckberg e St. Thomas) ou pinçamento intermitente. Foram feitas de uma a quatro pontes (média 2,8 ) por paciente (Tabela 1). A incisão cervical era suturada após neutralização da heparina.

Nos pacientes com obstrução carotídea bilateral, foi operado em primeiro lugar aquele com lesão mais acentuada ou de maior risco, por lesão ulcerada. Em 4 pacientes em que uma das carótidas internas estava ocluída, foi operada a artéria lesada porém pérvia, após o início da circulação extracorpórea com temperatura esofágica de $25^{\circ} \mathrm{C}$, para proteção cerebral.

Procurou-se, nesses pacientes, manter fluxo arterial adequado durante a circulação extracorpórea e pressão arterial média entre $50 \mathrm{mmHg}$ e $70 \mathrm{mmHg}$.

Endarterectomia foi realizada na carótida esquerda em 23 pacientes, na direita em 14 e bilateral em 9 .

\section{RESULTADOS}

Nesse grupo de operações associadas havia maior porcentagem $(34,7 \%)$ de pacientes com idade igual ou superior a 70 anos que o habitual do Serviço ${ }^{18}$, havia, também, maior incidência de hipertensão arterial sistêmica (HAS). A mortalidade imediata foi de $8,6 \%(4 / 46)$ e as causas dos óbitos foram:
TABELA 3

ENDARTERECTOMIA DE CARÓTIDA (EC) ASSOCIADA A REVASCULARIZAÇÃO MIOCÁRDICA (RM)

$N=46$

Causas de óbitos $-\mathrm{N}=4(8,6)$

Baixo Débito + Insuficiência Respiratória

Rotura Aorta Ascendente

Óbito durante Operação para

Revascularização de Membros Inferiores

IMO

baixo débito cardíaco associado a insuficiência respiratória; rotura da aorta ascendente no local da canulação (paciente foi reaberto e a correção foi realizada, mas, a partir daí, o paciente evoluiu com insuficiência renal e insuficiência respiratória) durante operação para realização de enxerto aortofemoral por isquemia grave de membro inferior no primeiro dia de pós-operatório da RM-EC; insuficiência de múltiplos órgãos (IMO) (Tabela 3). Dos 4 pacientes, 3 tinham 70 ou mais anos de idade. Nos 30 pacientes com menos de 70 anos de idade houve $1(3,3 \%)$ óbito (Tabela 4).

Não houve AVC permanente no pós-operatório; apenas 1 paciente apresentou parestesia transitória no lado não correspondente à endarterectomia. Nos 4 pacientes que tinham uma das carótidas ocluídas, a endarterectomia do lado oposto foi realizada com paciente em circulação extracorpórea e hipotermia sistêmica de $25^{\circ} \mathrm{C}$, não ocorrendo qualquer manifestação neurológica pós-operatória; acreditamos ser esta a melhor proteção cerebral nesses casos. Um paciente necessitou de balão intra-aórtico (BIA), por apresentar baixo débito cardíaco, evoluindo bem posteriormente; 1 paciente apresentou deiscência da sutura do esterno, sendo ressuturado, com bom resultado; 1 paciente apresentou hemorragia na linha de sutura da carótida, também corrigida com sucesso; 1 paciente necessitou de assistência ventilatória prolongada, com boa evolução.

A longo prazo (2 a 186 meses) (Tabela 5 e Gráfico 1) ocorreram 9 óbitos, 2 pacientes não foram localizados e 31 estão vivos. As causas dos óbitos

TABELA 4

ENDARTERECTOMIA DE CARÓTIDA (EC) ASSOCIADA A REVASCULARIZAÇĀO MIOCÁRDICA (RM)

\begin{tabular}{lccr}
\hline \multicolumn{4}{c}{ MORTALIDADE RELACIONADA COM IDADE } \\
\hline & $N$ & ÓBITOS & $\%$ \\
\hline Pacientes & 46 & 4 & 8,6 \\
Pacientes <70a & 30 & 1 & 3,3 \\
Pacientes $\geq 70 \mathrm{a}$ & 16 & 3 & 18,7 \\
\hline
\end{tabular}


SOUZA, J. M.; BERLINCK, M. F.; OLIVEIRA, P. A. F.; FERREIRA, R. P.; MAZZIERI, R.; OLIVEIRA, S. A. - Cirurgia de revascularização do miocárdio associada a endarterectomia de carótida. Rev. Bras. Cir. Cardiovasc., 10 (1): 43-49, 1995.

TABELA 5

ENDARTERECTOMIA DE CARÓTIDA (EC) ASSOCIADA A REVASCULARIZAÇÄO MIOCÁRDICA (RM)

\begin{tabular}{|c|c|c|}
\hline MÉDIA & os & $N=46$ \\
\hline Evolução & $N$ & $\%$ \\
\hline $\begin{array}{l}\text { Óbitos Imediatos } \\
\text { Óbitos Tardios } \\
\text { Nāo Localizados } \\
\text { Vivos }\end{array}$ & $\begin{array}{r}4 \\
9 \\
2 \\
31\end{array}$ & $\begin{array}{r}8,6 \\
19,5 \\
4,3 \\
67,3\end{array}$ \\
\hline
\end{tabular}

tardios foram: infarto agudo do miocárdio em 4 pacientes, 1 paciente faleceu no pós-operatório imediato de reoperação para RM e troca da valva aórtica e 1 por ICC; em 3 pacientes as causas foram: hemorragia digestiva em 1 , neoplasia em 1 e AVC em outro. Em resumo, 6 pacientes faleceram de causa cardíaca e 3 de causa não cardíaca.

O que necessita ser respondido é se a associação de doença aterosclerótica obstrutiva coronária e carotídea aumenta o risco cirúrgico do paciente. Se a indicação primária é a endarterectomia carotídea, o paciente terá maior risco de uma intercorrência cardíaca (infarto do miocárdio). Esse risco é baixo (1\%) nos pacientes assintomáticos do ponto de vista cardíaco, eleva-se para $7 \%$ nos pacientes sintomáticos (angina) e pode chegar a $17 \%$ naqueles com angina instável $4,8,11$. BERNHARD et alii ${ }^{4}$ operaram 31 pacientes com doença coronária e carotídea; em 15 pacientes foi feita endarterectomia de carótida inicialmente, com $5(33,3 \%)$ óbitos, sendo 3 por IAM; em 16 pacientes a cirurgia associada (RC+RM) foi sem mortalidade cirúrgica e com apenas um AVC transitório. Esses autores foram os primeiros a propor a cirurgia combinada. VERMEHLEN et alii ${ }^{19}$ relatam que $10 \%$ dos pacientes com indicação primária para EC têm doença cardíaca que justifica operação simultânea.

\section{GRÁFICO 1}

REVASCULARIZAČÃO MIOCÁRDICA ASSOCIADA A ENDARTERECTOMIA DE CARÓTIDA

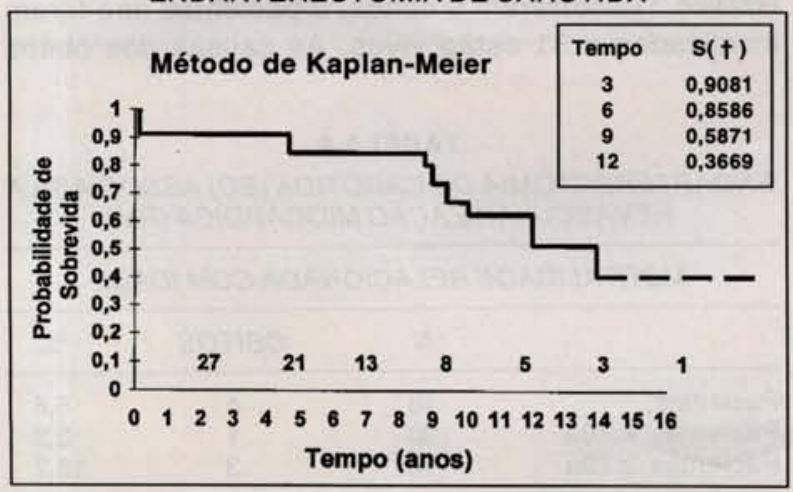

Para nós cirurgiões cardiovasculares o problema geralmente apresenta-se de maneira diferente; - paciente tem indicação para revascularização do miocárdio com doença obstrutiva de carótida associada. É muito importante definir corretamente a doença carotídea quanto à sintomatologia e quanto à lesão obstrutiva. Em muitos trabalhos encontramos referência apenas a sopro carotídeo; muitos pacientes têm sopro cervical sem obstrução de carótida, como mostram MEHIGAN et alii ${ }^{15}$, que fizeram arteriografia em 104 pacientes com sopro e oculopletismografia positiva encontrando obstruções significativas de uma ou de ambas carótidas em apenas 49 pacientes. Quanto à sintomatologia neurológica, existem muitos trabalhos mostrando que os pacientes com lesão de carótida e assintomáticos apresentam, baixo risco de AVC no pós-operatório de $R M 5,14,16,17$. Os pacientes com AVC prévio ou episódios de isquemia cerebral transitória têm elevada incidência de AVC trans-operatório ${ }^{6,9}$. quando a RM é feita isoladamente, principalmente quando a lesão carotídea é bilateral ${ }^{10}$, podendo chegar a $20 \%$ se uma das carótidas é ocluída 5 . Cirurgia combinada $(\mathrm{RM}+\mathrm{EC})$ está indicada nos pacientes que apresentam maior risco de infarto agudo do miocárdio se submetidos a EC isolada ou maior risco de AVC se submetidos a RM isolada; são pacientes mais graves que têm sintomatologia neurológica e cardiaca mais acentuada e lesões obstrutivas também mais significativas. Associação de obstrução carotídea é indicativo de doença coronária mais grave 16,17 - maior incidência de: lesão de tronco de coronária esquerda, doença triarterial, doença aterosclerótica da aorta ascendente, doença arterial periférica, comprometimento da função ventricular esquerda, hipertensão arterial sistêmica e pacientes mais idosos. JONES et alii ${ }^{14}$ compararam dois grupos de pacientes submetidos a RM, que tiveram ou não lesão neurológica pósoperatória. Os pacientes que tiveram lesão neurológica eram mais idosos, tinham maior incidência de sopro carotídeo e aorta ascendente com doença aterosclerótica mais acentuada.

JOHNSON et alii ${ }^{13}$, estudando fluxo cerebral em pacientes com obstrução carotídea durante CEC, não encontraram hipoperfusão, mas ALLEN ${ }^{1}$ comenta, em editorial, que esse dado não pode ser usado para diminuir a importância das obstruções carotídeas como fator de risco para lesão neurológica pós-operatória; a operação isolada ou combinada deverá ser decidida de acordo com cada caso.

SCHULTZ et alii ${ }^{17}$, revisando 1360 pacientes submetidos a RM concluíram; pacientes com doença carotídea assintomática podem ser submetidos a RM com CEC, sem risco adicional de AVC; mas esses pacientes têm maior risco de AVC a longo prazo. Associação de doença carotídea é sinal de 
SOUZA, J. M.; BERLINCK, M. F.; OLIVEIRA, P. A. F.; FERREIRA, R. P.; MAZZIERI, R.; OLIVEIRA, S. A. - Cirurgia de revascularização do miocárdio associada a endarterectomia de carótida.

Rev. Bras. Cir. Cardiovasc., 10 (1): 43-49, 1995.

maior gravidade, estando, portanto, associada à maior mortalidade cirúrgica e pior expectativa de vida.

GARDNER et alii ${ }^{10}$ estudaram 3279 pacientes submetidos a RM e observaram que o risco de AVC pós-operatório estava aumentado nos pacientes com idade mais avançada, com doença cerebrovascular no pré-operatório, com a duração acentuada do tempo de CEC, comprometimento aterosclerótico severo da aorta ascendente e hipotensão arterial grave trans-operatória.

RIZZO et alii 16 operaram, simultaneamente (EC+RM), 127 pacientes com mortalidade de 5,5\% ( $7 / 127)$ e incidência de AVC permanente em 7 (5,5\%) pacientes, sendo que em 5 o AVC ocorreu no lado operado. Observaram, também, pior sobrevida aos 5 anos de pós-operatório nos pacientes com função ventricular diminuída. Esses autores concluem: a cirurgia combinada $(R M+E C)$ é útil em pacientes de alto risco, evita reinternações, nova anestesia e protege os pacientes de AVC a longo prazo $(97 \%$ dos pacientes estavam livres de AVC com 8 anos de pós-operatório).

HETZER et alii ${ }^{12}$ trataram 129 pacientes assintomáticos com angina instável associada a lesão carotídea unilateral, de maneira randomizada (71 pacientes tiveram operação combinada $R M+E C$ e 58 pacientes RM inicialmente, seguida de EC posteriormente). A mortalidade operatória foi de $4,2 \%$ (3/71) e ocorreram $2(2,8 \%)$ AVC nos 71 pacientes com operação combinada; nos 58 pacientes com operações em dois tempos, $2(3,4 \%)$ pacientes faleceram após a RM e $1(1,9 \%)$ após a EC; ocorreram 8 AVC, sendo $4(7,5 \%)$ após a EC subseqüente. $A$ incidência total de $\mathrm{AVC}$ foi, portanto, $14 \%$, quando as operações foram realizadas separadamente.

A nossa experiência compara-se favoravelmente à literatura, quanto à ocorrência de complicações neurológicas, com apenas 1 paciente que apresentou hemiparesia contralateral à endarterectomia. Não utilizamos shunt interno; em todos os casos foi possivel fazer endarterectomia adequada com tempo de pinçamento rápido. Acreditamos que a utilização de CEC com hipotermia profunda, nos paci- entes com lesão carotídea de um lado e oclusão do outro foi efetiva na proteção cerebral. Outros autores tiveram, em pacientes com essa associação, uma elevada incidência de AVC trans-operatório.

A operação combinada $(R M+E C)$, além de diminuir a incidência de AVC no pós-operatório imediato, principalmente nos pacientes com lesão carotídea bilateral e naqueles com passado de AVC ou isquemia cerebral transitória, tem também como objetivo proteger os pacientes a longo prazo da ocorrência de AVC; dos nossos pacientes operados, 1 apresentou AVC a longo prazo; esse paciente tinha lesão bilateral e foi operado apenas de um lado.

Tivemos mortalidade operatória de $8,6 \%$, sendo que dos 4 óbitos, 3 ocorreram em pacientes com 70 ou mais anos de idade e em nenhum paciente 0 óbito foi atribuído à combinação de operações. O aumento do tempo para realização deste tipo de operação não influencia a mortalidade ou a morbidade. Os benefícios são: menor incidência de complicações neurológicas, menor custo, hospitalização e anestesia únicas e proteção a longo prazo de complicações neurológicas.

Utilizamos sempre a anestesia geral; não há qualquer benefício na utilização de anestesia local ou regional, para o tempo da endarterectomia, como preconizado por alguns cirurgiões, além de desconfortável e "estressante", o que é prejudicial, principalmente para pacientes com lesões coronarianas acentuadas, condiçōes hemodinâmicas instáveis e função ventricular deprimida. Acreditamos que a operação combinada está indicada em grupo especial de pacientes; em nosso Serviço ela foi indicada em $0,46 \%$ dos pacientes $(46 / 10940)$ submetidos a revascularização do miocárdio.

A ocorrência de AVC trans-operatório depende de uma série de fatores, como idade avançada, doença aterosclerótica da aorta ascendente, tempo prolongado de CEC, hipotensão arterial e AVC prévio, sendo que certamente os pacientes com lesões carotídeas bilaterais ou lesões suboclusivas apresentam maior risco de complicação neurológica trans-operatória. 
SOUZA, J. M.; BERLINCK, M. F.; OLIVEIRA, P. A. F.; FERREIRA, R. P.; MAZZIERI, R.; OLIVEIRA, S. A. - Cirurgia de revascularização do miocárdio associada a endarterectomia de carótida. Rev. Bras. Cir. Cardiovasc., 10 (1): $43-49,1995$.

RBCCV $44205-257$

SOUZA, J. M.; BERLINCK, M. F.; OLIVEIRA, P. A. F.; FERREIRA, R. P.; MAZZIERI, R.; OLIVEIRA, S. A. Combined operation for myocardial revascularization and carotid endarterectomy. Rev. Bras. Cir. Cardiovasc., 10 (1): 43-49, 1995.

ABSTRACT: There are still controversies about the treatment of associated coronary artery disease and carotid artery obstruction. Between 1979 and 1994, 10940 patients were operated on for myocardial revascularization. Combined operations (myocardial revascularization and carotid endarterectomy) were done in $46(0.43 \%)$ patients, during the same period. Patients age ranged from 48 to 76 years with an average of $65.2 \mathrm{yrs} ; 80.4 \%$ were male; 23 had had previous myocardial infarction. Associated diseases were diabetes mellitus in 10 patients, chronic renal insufficiency in $5 ; 29$ were in functional class III or IV for angina; 4 patients had congestive cardiac insufficiency: left main carotid obstruction, and in 4 of them one carotid artery was completaly obstructed; 23 patients had had transient cerebral ischemic attack and 2 had had stroke with sequelae. Hospital mortality was $8.6 \%$ (4/46). Permanent stroke did not occur in the operative period; 3 of the 4 deaths occurred in patients 70 years or older. Carotid endarterectomy was done just before cardiopulmonary bypass in 42 patients and in 4 patients with one of the carotid arteries occluded, done after cardiopulmonary bypass was established and the patient temperature was $25^{\circ} \mathrm{C}$. Considering that no patient had perioperative stroke, we think that this strategy is adequate for this association of diseases.

DESCRIPTORS: Myocardial revascularization, surgery. Carotid artery, endarterectomy.

\section{REFERÊNCIAS BIBLIOGRÁFICAS}

1 ALEN, B. T. - Cardiopulmonary perfusion and cerebral blood flow in bilateral carotid disease. Ann. Thorac. Surg., 51: 532-533, 1991.

BARNES, R. W.; MARSZALEK, P. B.; RITTGERS, S. E. - Asymptomatic carotid disease in preoperative patients. Stroke, 11: 136-139, 1980.

BARES, R. W.; NIX, M. L.; SANSONETTI, D.; TURLEY, G.; GOLDMAN, M. R. - Late outcome of untreated asymptomatic carotid disease following cardiovascular operations. J. Vasc. Surg., 2: 843-849, 1985.

4 BERNHARD, V. M.; JOHNSON, W. D.; PETERSON, J. J. - Carotid artery stenosis - association with surgery for coronary artery disease. Arch. Surg., 105: 837$840,1972$.

5 BRENER, B. J.; BRIEF, D. K.; ALPERT, J.; GOLDENKRANZ, R. J.; PARSONETT, V. - The risk of stroke in patients with asymptomatic carotid stenosis undergoing cardiac surgery: a follow-up study. J. Vasc. Surg., 5: 269-279, 1987.

COSGROVE, D. M.; HERTZER, N. R.; LOOP, F. D. Surgical management of synchronous carotid and coronary artery disease. J. Vasc. Surg., 4: 690$697,1986$.
7 EMERY, R. W.; COHN, L. J.; WHITTEMOREE, A. D.; MANNICK, J. A.; CONCH, A.; COLLINS, J. J. Coexistent carotid and coronary artery disease. Arch Surg., 118: 1035-1038, 1983.

8 ENNIX Jr., C. L.; LAWRIE, G. M.; MORRIS Jr., G. C.; CRAWFORD, E. S.; HOWELL, J. F. L.; REARDON, J. J.; WEATHERFORD, S. C. - Improved results of carotid endarteerectomy in patients with symptomatic coronary disease: an analysis of 1.546 consecutive carotid operations. Stroke, 10: 122-125, 1979.

9 FAGGIOLI, G. L.; CURL, R.; RICOTTA, J. J. - The role of carotid screening before coronary artery bypass. J. Vasc. Surg., 12: 724-731, 1990.

10 GARDNER, T. J.; HORNEFFER, P. I.; MANOLIO, T. A.; PEARSON, T. A.; GOTT, V. L.; BAUMGARTNER, W. A.; BORKON, M.; WATKINS, L.; REITZ, B. A. Stroke following coronary artery bypass. Ann. Thorac. Surg., 40: 574-581, 1985.

11 HERTZER, N. R. \& LEEDS, D. C. - Fatal myocardial infarction following carotid endarterectomy. Ann. Surg., 194: 212-218, 1981.

12 HERTZER, N. R.; LOOP, F. D.; BEVEN, E. G.; O'HARA, P. J.; KRAJEWSKI, L. P. - Surgical staging for simultaneous coronary and carotid disease: a study including prospective randomization. J. Vasc. Surg., 9: 455-463, 1991. 
SOUZA, J. M.; BERLINCK, M. F.; OLIVEIRA, P. A. F.; FERREIRA, R. P.; MAZZIERI, R.; OLIVEIRA, S. A. - Cirurgia de revascularização do miocárdio associada a endarterectomia de carótida. Rev. Bras. Cir. Cardiovasc., 10 (1): $43-49,1995$.

13

JOHNSON, P.; ALGOTSSON, L.; RYDING, E.; STAHL, E.; MESSETER, K. - Cardiopulmonary perfusion and cerebral blood flow in bilateral carotid disease. Ann. Thorac. Surg., 51: 579-584, 1991.

14 JONES, E. L.; CRAVER, J. M.; MICHALIK, R. A.; MURPHY, D. A.; GUYTON, R. A.; BONE, D. K.; HATCHER, C. R.; REICHAWALD, N. A. - Combined carotid and coronary operations: when are they necessary? J. Thorac. Cardiovasc. Surg., 87: 716,1984 .

15 MEHIGAN, J. T.; BUCH, W. S.; PIPKIN, R. D.; FOGARTY, T. J. - A planned approach to coexistent cerebrovascular disease in coronary artery bypass candidates. Arch. Surg., 112: 1403-1409, 1977.

16 RIZZO, R. J.; WHITTEMORE, A. D.; COUPER, G. S.; DONALDSON, A.; ARANKY, S. F.; COLLINS, J. J.; MUNNICK, J. A.; COHN, L. H. - Combined carotid and coronary revascularization: the preferred approach to the severe vasculopathy. Ann. Thorac. Surg., 54: 1099-1109, 1992.

SCHULTZ, R. D.; STERPETH, A. V.; FELDHAUS, R. J. - Early and late results in patients with carotid disease undergoing myocardial revascularization. Ann. Thorac. Surg., 45: 603-609, 1988.

18 SOUZA, J. M.; BERLINCK, M. F.; MOREIRA, M. G.; MARTINS, J. R. M.; MOURA, M. C.; OLIVEIRA, P. A. F.; SENRA, D. F.; OLIVEIRA, S. A. - Revascularização miocardica em pacientes com idade igual ou superior a 70 anos. Rev. Bras. Cir. Cardiovasc., 5: $141-148,1990$.

19 VERMEHLEN, F. E. E.; HAMERLIJNCK, P. H. M.; DEFAUW, J. J. A. M.; ERNEST, S. M., P. G. Synchronous operation for ischemic cardiac and cerebrovascular disease: early results and longterm follow-up. Ann. Thorac. Surg., 53: 381-390, 1992. 